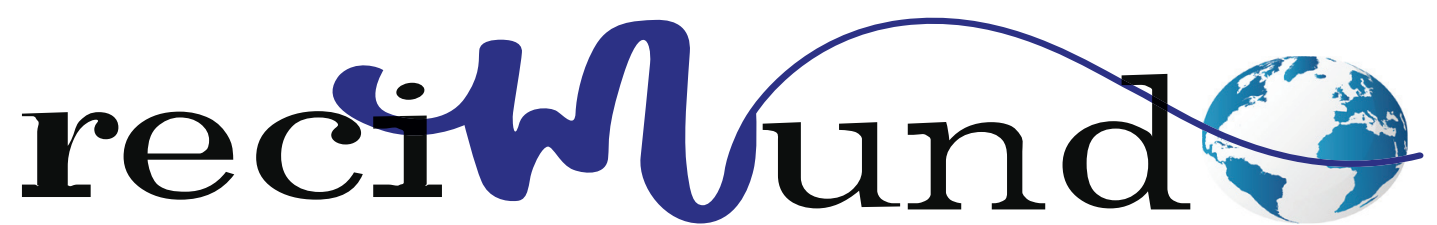

Revista Científica Mundo de la Investigación y el Conocimiento

DOI: $10.26820 /$ recimundo/5.(1).enero.2021.20-31

URL: http://recimundo.com/index.php/es/article/view/979

EDITORIAL: Saberes del Conocimiento

REVISTA: RECIMUNDO

ISSN: 2588-073X

TIPO DE INVESTIGACIÓN: Artículo de Revisión

CÓDIGO UNESCO: 3205 Medicina Interna

PAGINAS: $20-31$

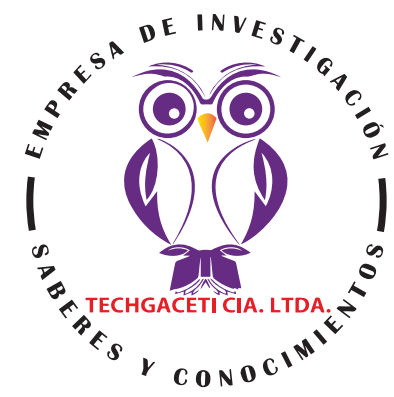

\title{
Características hematológicas en pacientes con diabetes mellitus tipo 2
}

Hematologic characteristics in patients with type 2 diabetes mellitus

Características hematológicas em pacientes com diabetes mellitus tipo 2

María José Campoverde Maldonado; Javier Alejandro Rosero Caiza²; Germán Esteban Rosero Caiza;

Michelle Estefanía Benavides Arteaga ${ }^{4}$

\section{RECIBIDO: 28/11/2020 ACEPTADO: 06/12/2020 PUBLICADO: 31/01/2021}

1. Especialista en Salud y Seguridad Ocupacional; Médico; Investigador Independiente; Cuenca, Ecuador; majocampoverde@hotmail.es; (D) https://orcid.org/0000-0003-1496-994X

2. Médico Cirujano de la Pontificia Universidad Católica del Ecuador; Investigador Independiente; Quito, Ecuador; javier. rosero94@gmail.com; (iD https://orcid.org/0000-0002-3635-6653

3. Médico Cirujano de la Pontificia Universidad Católica del Ecuador; Investigador Independiente; Quito, Ecuador; germanesteban32@gmail.com; iD https://orcid.org/0000-0001-9655-2747

4. Médica Cirujana de la Universidad de las Américas; Investigador Independiente; Quito, Ecuador; mebenavides.3@hotmail. com; (D) https://orcid.org/0000-0002-4099-6151

\section{CORRESPONDENCIA}

María José Campoverde Maldonado

majocampoverde@hotmail.es

Cuenca, Ecuador

(C) RECIMUNDO; Editorial Saberes del Conocimiento, 2021 


\section{RESUMEN}

Cuando se habla de la diabetes mellitus de tipo 2 (DM 2/ DT 2) se hace referencia a un trastorno crónico (incurable, pero controlable) que perturba la forma en la cual el cuerpo metaboliza la glucosa (azúcar), una fuente importante de energía para el organismo. El objetivo que se pretende alcanzar mediante el desarrollo del presente estudio es el de compendiar criterios expertos respecto a la caracterización hematológica en pacientes con DM 2; es por ello que se ha planeado enmarcar la investigación a un diseño bibliográfico y a una metodología de revisión, con la cual se expondrá sobre: definiciones fundamentales de DM 2, su etiología, factores de riesgo y algunas de sus características hematológicas en los pacientes que la padecen. En los resultados se evidencia el alcance de los objetivos propuestos, incluso se logra referir algunos estudios representativos de los que se extraen los valores referenciales de algunos componentes del perfil hematológico en la DM 2. Fue posible concluir que, en pacientes con DM 2, es hematológicamente característico encontrar alteraciones, principalmente, en los valores de: Insulina (elevada); Glucosa o Glucemia (elevada), HbA1c (elevada), media de hematocritos (disminuida), recuento de monocitos (elevados), porcentaje promedio de linfocitos (reducidos), PCR y transaminasas TGO (elevadas), colesterol (elevado), triglicéridos (elevados); así como probablemente pueden estar particularmente alteradas otras variables menos frecuentes.

Palabras clave: Resietencia insulinica, hemoglobina glicosilada, hiperglucemia, hematocritos, transaminasas TGO.

\section{ABSTRACT}

When talking about type 2 diabetes mellitus (DM 2 / TD 2) it refers to a chronic disorder (incurable, but controllable) that disturbs the way in which the body metabolizes glucose (sugar), an important source of energy for the body. The objective to be achieved through the development of this study is to summarize expert criteria regarding hematological characterization in patients with DM 2; That is why it has been planned to frame the research to a bibliographic design and to a review methodology, with which it will be discussed: fundamental definitions of DM 2, its etiology, risk factors and some of its hematological characteristics in patients who undergo it. suffer. The results show the achievement of the proposed objectives, it is even possible to refer some representative studies from which the reference values of some components of the hematological profile in DM 2 are extracted. It was possible to conclude that, in patients with DM 2, it is Hematologically characteristic, find alterations, mainly, in the values of: Insulin (high); Glucose or Glycemia (high), HbA1c (high), mean hematocrit (low), monocyte count (high), average percentage of lymphocytes (low), CRP and TG transaminases (high), cholesterol (high), triglycerides (high)); as well as other less frequent variables may be particularly altered.

Keywords: Insulin resistance, glycosylated hemoglobin, hyperglycemia, hematocrit, TGT transaminases.

\section{RESUMO}

Quando se fala de diabetes mellitus tipo 2 (DM 2 / TD 2) refere-se a uma desordem crônica (incurável, mas controlável) que perturba a forma como o corpo metaboliza a glicose (açúcar), uma importante fonte de energia para o organismo. 0 objetivo a ser alcançado através do desenvolvimento deste estudo é resumir os critérios de especialistas em caracterização hematológica em pacientes com DM 2; por isso foi planejado enquadrar a pesquisa em um desenho bibliográfico e em uma metodologia de revisão, com a qual será discutida: definições fundamentais de DM 2, sua etiologia, fatores de risco e algumas de suas características hematológicas em pacientes que sofrem. Os resultados mostram a realização dos objetivos propostos, é até possível referir alguns estudos representativos dos quais são extraídos os valores de referência de alguns componentes do perfil hematológico em DM 2. Foi possível concluir que, em pacientes com DM 2, é hematologicamente característico, encontrar alterações, principalmente, nos valores de: Insulina (alta); Glicose ou Glicemia (alta), HbA1c (alta), hematócrito médio (baixo), contagem de monócitos (alta), percentual médio de linfócitos (baixo), CRP e TG transaminases (alto), colesterol (alto), triglicérides (alto) ); assim como outras variáveis menos freqüentes podem ser particularmente alteradas.

Palavras-chave: Resistência à insulina, hemoglobina glicosilada, hiperglicemia, hematócrito, transaminases TGT. 
M. E.

\section{Introducción}

La Federación Internacional de la Diabetes (IDF, por sus siglas en inglés) se refiere a la diabetes mellitus (DM), también conocida simplemente por el termino de diabetes, como:

una afección grave y de largo plazo (o "crónica") que ocurre cuando los niveles de glucosa en la sangre de una persona son altos porque su cuerpo no puede producir insulina o la cantidad suficiente de esta hormona, o cuando no puede utilizar de manera eficaz la insulina que produce. (IDF, 2019)

Por su parte, Brutsaert (2019) destaca que la diabetes mellitus (DM) "se debe a la secreción anormal de insulina y a grados variables de resistencia periférica a la insulina, que conducen a la aparición de hiperglucemia"

Cuando se habla de la diabetes mellitus de tipo 2 (DM 2/ DT 2) se hace referencia a un trastorno crónico (incurable, pero controlable) que perturba la forma en la cual el cuerpo metaboliza la glucosa (azúcar), una fuente importante de energía para el organismo. En otras palabras, puede darse el caso de que el cuerpo (los adipocitos, los hepatocitos y las células musculares) generan resistencia a los efectos de la insulina (hormona que regula el desplazamiento del azúcar en las células) lo que significa que el azúcar de la sangre no entra en dichas células con el fin de ser almacenada como fuente de energía; o que, en caso distinto, el propio organismo no produce suficiente insulina para reservar la glucosa en sus niveles normales. (Mayo Clinic - USA, 2020; MedlinePlus, 2020)

Los pacientes con diabetes mellitus tipo 2 (DM 2) representan el 90\% de los casos de diabetes en todo el mundo, y su prevalencia (ver Figura 1), además de estar bastante elevada, todavía sigue en aumento (IDF, 2019)

Este aumento se debe al envejecimiento de la población, al desarrollo económico y al incremento de la urbanización, lo que deriva en estilos de vida más sedentarios e incremento en el consumo de alimentos poco saludables que se relacionan con la obesidad. (IDF, 2019, pág. 14)

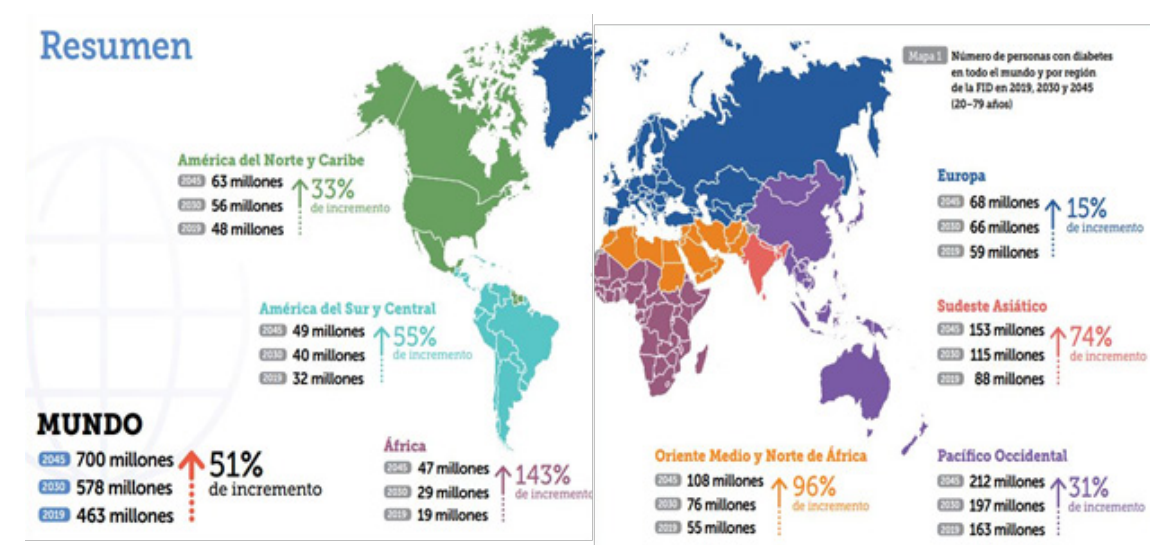

Figura 1. Resumen global de personas entre 20 y 79 diagnosticadas con Diabetes

NOTA: La figura representa las diferentes regiones a nivel mundial con sus respectivas números de personas que padecen DM, detallando el calculo resgistrado hasta el 2019 y sus estimaciones de incremento en el número de personas enfermas con esta misma patología para 2030 y 2045, indicando igualmente su porcentaje de aumento. Tomado de Atlas de la Diabetes de la FID 2019 (pp. 4-5) por FID, 2019, Federación Internacional de Diabetes. 
La DM 2 también resulta ser una de las primeras causas de enfermedad temprana y de muerte. Su prevalencia en adultos se estima en un 6,4\%, y dependiendo de la región, puede oscilar entre 3,8\% y 10,2\%; sin embargo, en algunas zonas las tasas de diabetes no detectada pueden llegar hasta un 50\%. (Parra \& Martínez, 2019)

La DM2 ha mostrado una progresiva transición epidemiológica, principalmente por adopción de malos hábitos en el estilo de vida. Reflejo de lo anterior, se evidencia en Latinoamérica donde se ha venido presentando un aumento en la prevalencia y mortalidad, estudios que incluyen grandes ciudades como México, Bogotá y Santiago de Chile, estipulan valores de prevalencia tan altos como $8.9,8.1$ y $7.2 \%$ respectivamente. Estudios recientes han mostrado a México y Brasil como los países latinoamericanos que están dentro de los primeros diez países con mayor número de pacientes con DM2 a nivel mundial. (Osuna et al, 2014)

Otro aspecto importante también destacado por la IDF se refiere a que todavía se ha encontrado una explicación completa a las causas de la DM 2, no obstante, dejan ver que sí hay una relación bastante angosta con el sobrepeso, la obesidad y la edad madura, así como también con el origen étnico y ciertos antecedentes familiares. El origen de la DM 2, de la misma manera que en la diabetes tipo 1, surge por la conjunción de una predisposición multigénica y desencadenantes ambientales. (IDF, 2019, pág. 14)

Los síntomas de la DM 2 son bastante parecidos a los de diabetes tipo 1, sin embargo, comúnmente se manifiesta de forma menos severa, incluso, es posible que ni siquiera se perciban sus síntomas durante muchos años. También es posible que los primeros síntomas en la DM 2 son propios de las complicaciones de la diabetes, cuadro que entonces sugiere que dicha patología ha venido evolucionando desde hace tiempo considerable (Brutsaert, 2019) Entre los síntomas más comunes se encuentran: cansancio (fatiga), aumento de las ganas de comer o tomar agua, incremento de la micción (necesidad de orinar o en su volumen, pérdida de peso, visión borrosa, retardo en la sanación de heridas, infección en la vejiga, el riñón, la piel u otras infecciones que son más frecuentes. (MedlinePlus, 2020)

De conformidad a lo publicado por el Instituto Nacional de Diabetes y Enfermedades Digestivas y Renales (NIDDK, por sus siglas en inglés) (2016), para detectar la diabetes tipo 2 (DM 2), la recomendación de los expertos es realizar las pruebas de rutina si: a) la edad de la persona es superior a los 45 años, b) si la edad de la misma se ubica entre los 19 y 44 años, pero tiene sobrepeso u obesidad y presenta uno o más factores de riesgo de diabetes, o c) si se trata de una mujer que ha tenido diabetes gestacional.

"Aunque la diabetes tipo 2 se presenta con mayor frecuencia en los adultos, también se puede presentar en los niños" (NIDDK, 2016) razón por la que los especialistas advierten que, deben hacerse las pruebas de detección de diabetes en niños y adolescentes entre los 10 y 18 años de edad, cuando padecen sobrepeso u obesidad y, por lo menos dos, de los siguientes factores de riesgo: si tuvo bajo peso al nacer (BPN); si es hijo de madre que padeció diabetes durante su embarazo (gestacional) o si aplica en cualquier otro de los factores de riesgo conocidos propiamente para la DM 2, tales como: sobrepeso u obesidad; mayor de 45 años de edad; con antecedentes familiares de diabetes; de descendencia étnica afroamericana, hispana/latina, Islas del Pacífico, nativo de Alaska, indígena estadounidense, estadounidense de origen asiático o nativo de Hawái; presión arterial alta; bajo nivel de colesterol HDL o un nivel alto de triglicéridos; antecedentes de diabetes gestacional o si dio a luz a un hijo con más de 4 kilogramos (9 libras); inactividad física; si posee antecedentes de enfermedades cardíacas o cardiovasculares; depresión;

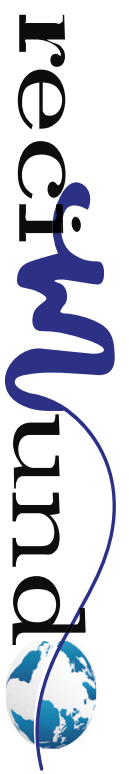


M. E.

síndrome de ovario poliquístico o zonas de piel alrededor del cuello o las axilas oscurecidas, gruesas y aterciopeladas (acantosis nigricans).

Ahora bien, el objetivo que se pretende alcanzar mediante el desarrollo del presente estudio es el de compendiar criterios expertos respecto a la caracterización hematológica en pacientes con DM 2. En base a ello, se ha planeado enmarcar la investigación a un diseño bibliográfico y a una metodología de revisión, con la cual se expondrá sobre: definiciones fundamentales, etiología, factores de riesgo y algunas características hematológicas en pacientes diagnosticados con DM 2.

A continuación, se expone sobre la metodología investigativa aplicada, para proseguir con la sección los resultados, donde se evidenciarán el contenido más específico en torno a la temática planteada y finalmente concluir en base a tales fuentes de información.

\section{Materiales y métodos}

El desarrollo de esta revisión bibliográfica se ha hecho bajo un modelo de investigación documental, y a tales fines se hace indispensable comenzar con la búsqueda de información mediante la utilización de material bibliográfico disponible en forma física y digital; tales como libros, revistas especializadas, artículos científicos, boletines, notas científicas descriptivas, folletos, tesis de profesionalización y estudios superiores, datos estadísticos oficiales, entre otros. Cabe resaltar que el acceso a las mencionadas fuentes físicas fue posible mediante el uso de la biblioteca de la propia Casa de Estudios. Los recursos digitales pudieron hallarse mediante el uso de la internet, por medio de consultas en algunas bases de datos, como las de: la Biblioteca Nacional de Medicina (NLM, por sus siglas en inglés) y NIDDK, ambos entes con sede en los EE.UU. de América; la del Portal Re- gional de la Biblioteca Virtual de la Salud (BVS); SciELO; Medigraphic; REDIB, Google Scholar, y otras. De la misma manera se buscó en portales web de organismos oficiales nacionales e internacionales, por ejemplo, en el del Ministerio de Salud Pública del Ecuador (MSPE), el de la Organización Mundial de la Salud (OMS/WHO, por sus siglas en inglés), el de la Organización Panamericana de la Salud (OPS/PAHO, por sus siglas en inglés), entre otras.

La búsqueda se caracterizó y estuvo delimitada al uso de palabras y expresiones claves junto con operadores lógicos y booleanos, con los cuales fue posible construir algunas formulaciones para la exploración, tales como:

- características hematológicas AND paciente con diabetes mellitus tipo 2.

- características hematológicas AND paciente diabético tipo 2 OR DM 2 OR DT 2

- $\quad$ perfil hematológico +DM 2|DT 2.

- $\quad$ valores hematológicos +DM 2|DT 2.

Los resultados obtenidos fueron muy pocos y ello se cree que fue por la especificidad con la que se hicieron las pesquisas mediante las formulaciones antes descritas, aspecto que no se intentó modificar dado que lo que se persigue con preferencia solo es información relacionada con las características hematológicas de pacientes con diabetes tipo 2.

Entre todo el material examinado de manera física y digital, se prestó especial atención a los artículos científicos originales, revisiones sistemáticas, casos (reportes) clínicos, actas o resúmenes de conferencias, tesis de grado, postgrado y doctorado, libros y secciones de libros, publicaciones médicas de la especialidad (nefrología, endocrinología, bioanálisis), y otros; que junto al estudio de las otras publicaciones de organismos, instituciones, asociaciones y demás entes competentes halladas en sus propios sitios web, fue posible lograr la ordenación, análi- 
sis, resumen y discusión entre cada uno de los miembros del equipo investigador, sin que hubiera lugar a ningún conflicto de intereses entre los mismos.

El compendio bibliográfico definitivamente seleccionado para la presente entrega sustenta todas las ideas y argumentos expresadas por el equipo investigador, y al mismo tiempo sirve para proveer nuevas bases teórico-científicas de futuras investigaciones en torno al tema en cuestión.

\section{Resultados}

Ante todo, en la diabetes tipo 2, la hiperglucemia es el resultado de la incapacidad de las células del cuerpo de responder totalmente a la insulina, lo que se conoce como "resistencia a la insulina". Durante el estado de resistencia a la insulina, la hormona no es eficaz, lo que deriva en un aumento de la producción de insulina. Con el tiempo, se puede llegar a una producción de insulina inadecuada (Figura 2) porque las células beta pancreáticas no cumplen con la demanda. (IDF, 2019)

Conforme a la obra de Empendium (2020) que se titula: manual de Medicina Interna Basada en la Evidencia (Manual MIBE) se refiere que, la DM 2:

Se produce por la alteración progresiva de la secreción de insulina en condiciones de insulinorresistencia. Se reconocen también otros mecanismos patogénicos en su desarrollo como la elevación del glucagón y del umbral renal de excreción de glucosa, o la disminución del efecto incretina. Puede estar condicionada genéticamente (herencia poligénica), pero la edad y factores ambientales desempeñan un papel decisivo (obesidad abdominal y escasa actividad física). El exceso de ácidos grasos libres, liberados por el tejido adiposo, es responsable de la lipotoxicidad, es decir un exceso de oxidación de las grasas que en el músculo inhibe la glucólisis, mientras que en el hígado favorece la gluconeogénesis. Para contrarrestar ambos procesos se requiere un aumento compensador de la secreción de insulina que puede provocar en personas susceptibles, el agotamiento gradual de las células $\beta$ y dar lugar a la elevación de la glucemia. Una vez presente la hiperglucemia, esta provoca la glucotoxicidad, la que sumada a la lipotoxicidad deteriora aún más la capacidad secretora de la insulina y la sensibilidad a la misma.

Por su parte, Brutsaert (2019) precisa la etiología de ésta patología, al describir que: En la diabetes mellitus tipo 2 (antes conocida como del adulto o no dependiente de la insulina), la secreción de insulina es inadecuada porque los pacientes han desarrollado resistencia a la insulina. La resistencia hepática a la insulina inhibe la supresión de la producción de glucosa hepática, y la resistencia periférica a la insulina afecta la absorción periférica de glucosa. Esta combinación da lugar a la hiperglucemia en ayunas y posprandial. Los niveles de insulina a menudo son muy altos, especialmente al principio de la enfermedad. Más tarde en el transcurso de la enfermedad, la producción de insulina puede caer, lo que exacerba la hiperglucemia.

En general, la enfermedad aparece en adultos y es más frecuente a medida que avanza la edad; hasta un tercio de los adultos > 65 años de edad tienen tolerancia alterada a la glucosa. En los adultos mayores, los niveles de glucemia alcanzan niveles más altos después de la ingesta que en los adultos más jóvenes, especialmente después de comidas con alta carga de carbohidratos. Los niveles de glucosa también tardan más en retornar a valores normales, en parte como consecuencia de la acumulación de grasa visceral y abdominal y la disminución de la masa muscular.

... Se identificaron determinantes genéticos, reflejados en la prevalencia elevada de la enfermedad 
M. E.

en algunos grupos étnicos (en especial, indoamericanos, latinos y asiáticos) y en familiares de los pacientes. Aunque se detectaron algunos polimorfismos genéticos durante los últimos años, no se haIló un solo gen responsable de las formas más frecuentes de DM tipo 2.

La patogenia es compleja y mal comprendida. Aparece una hiperglucemia cuando la secreción de insulina ya no puede compensar la resistencia a la insulina. Aunque la resistencia a la insulina es característica de los pacientes con DM tipo 2 y en aquellos que se encuentran en riesgo de desarrollar esa enfermedad, también existe evidencia de una disfunción en las células beta y un deterioro en la secreción de insulina, incluso un deterioro en la primera fase de la secreción de insulina en respuesta a la infusión intravenosa de glucosa, una pérdida de la secreción normalmente pulsátil de insulina, un incremento en la secreción proinsulínica que marca un deterioro en el procesamiento de insulina y una acumulación de polipéptido amiloide (una proteína que en, condiciones normales, se secreta junto con la insulina). Por sí sola, la hiperglucemia puede deteriorar la secreción de insulina porque las dosis altas de glucosa desensibilizan a las células beta o causan una disfunción de las células beta (toxicidad de la glucosa). Normalmente, estos cambios llevan años en presencia de una resistencia a la insulina.

La obesidad y el aumento de peso son determinantes sustanciales de la resistencia a la insulina en la DM tipo 2. Ambos se asocian con algunos determinantes genéticos, pero también reflejan el impacto de la dieta, el ejercicio y el estilo de vida. La incapacidad para suprimir la lipólisis en el tejido adiposo incrementa las concentraciones plasmáticas de ácidos grasos libres, que pueden comprometer el transporte de glucosa estimulado por la insulina y la actividad de la glucógeno sintasa muscular. El tejido adiposo también parece funcionar como un órgano endocrino que libera múltiples factores (adipocitocinas) capaces de influir de manera favorable (adiponectina) y desfavorable (factor de necrosis tumoral-alfa, IL-6, leptina, resistina) sobre el metabolismo de la glucosa. La restricción del crecimiento intrauterino y el bajo peso al nacer también se asociaron con resistencia a la insulina a una edad más avanzada y pueden reflejar las influencias ambientales prenatales adversas sobre el metabolismo de la glucosa. (Brutsaert, 2019)

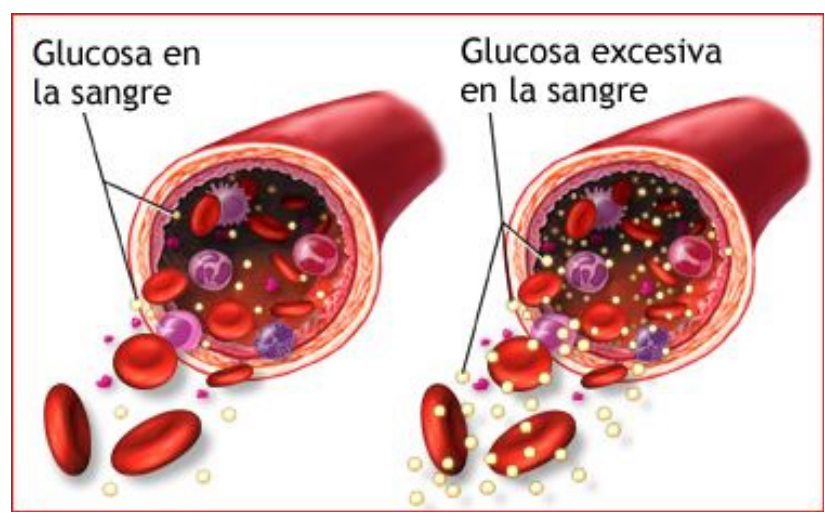

Figura 2. La Glucosa en sangre

NOTA: La figura representa la diferencia entre una concentración normal y excesiva de glucosa en la sangre. Tomado de Glucosa en la sangre (Página Web) por MedlinePlus, 2020, A.D.A.M. Inc.

\section{Factores de riesgo}

De acuerdo a algunas fuentes especializadas, los siguientes factores constituyen un incremento de riesgo de padecer DM 2:

- Edad.

- El peso.

- La distribución de la grasa.

- Inactividad física.

- Antecedentes familiares (susceptibilidad genética).

- Etnia.

- Prediabetes.

- Diabetes gestacional.

- Síndrome de ovario poliquístico. 
- Zonas de piel oscurecida.

- Fármacos.

- Tabaquismo.

- Otros. (Ministerio de Salud Publica de Uruguay, 2017; Mayo Clinic - USA, 2020; Valer, 2020)

Conforme al objetivo preestablecido para este estudio, es importante aclarar que, hacer referencia a las características hematológicas en pacientes con DM 2, significa aproximarse a la descripción de un perfil que se puede construir en base a los análisis de sangre de tales. Es por ello que, en lo sucesivo, se procura efectuar una descripción, fundamentalmente, de los valores referenciales que se han de obtener conforme al fundamento teórico-científico hallado, o bien se encontrasen reportados en estudios particulares donde se describan, caractericen o perfilen hematológicamente pacientes con DM 2, tras el análisis de sus muestras sanguíneas realizado mediante las pruebas o exámenes de laboratorio que corresponden en tales casos clínicos.

Antes de iniciar abordar la idea antes expuesta, se ha considerado didáctico previamente definir básicamente, cuáles son esas pruebas o exámenes propios de la DM 2 con los que bien se pudiera definir una caracterización hematológica. En este sentido, varias de las fuentes consultadas indican que pueden ser:

1. Glucemia.

- En ayunas.

- Prueba de tolerancia oral a la glucosa (PTGO / OGTT, por sus siglas en inglés)

2. Insulina (en ayunas, sérica, total o libre).

3. Hemoglobina Glicosilada (Glicada o $\mathrm{HbA1c}$ ).

4. Fructosamina.

5. Autoanticuerpos contra proteínas de la célula $\beta$.

6. Concentración del péptido $\mathrm{C}$ en el suero.

7. Concentración de cuerpos cetónicos en sangre.

8. Concentración de ácido láctico en suero.

9. Prueba de estímulo con glucagón.

10. Estimación de la sensibilidad a la insulina con el método HOMA.

11. Otros. (NIDDK, 2016; Abbott, 2017; Mayo Clinic - USA, 2020; MedlinePlus, 2020; Brutsaert, 2019; Empendium, 2020)

\section{Importancia de la Hemoglobina Glicosila- da (Glicada o HbA1c)}

Dada la trascendencia de ésta variable en particular, se ha considerado ampliar otros aspectos que han sido destacado en la mayoría de las fuentes consultadas.

El estudio de ésta valor hematológico puede ser considerado como un marcador principal (aunque no el único) de control glucémico de la diabetes mellitus, incluso su variabilidad (estudio de las aproximaciones de la glucosa hacia hiperglucemia como hacia la hipoglucemia) también pudiera asumirse como un marcador de complicaciones crónicas de la diabetes mellitus. (Suarez, 2019)

Jiménez, en términos sencillos, explica que con esta prueba se trata de evaluar retrospectivamente el control de la diabetes, en otras palabras, la media de los niveles de glucosa en sangre al menos durante los últimos 3 meses.

Los glóbulos rojos que circulan por la sangre contienen una proteína llamada hemoglobina. La glucosa, que también circula por la sangre, se adhiere a la hemoglobina durante un periodo de entre 90 y 120 días (aproximadamente 3 meses). De esta manera, la prueba de la hemoglobina glicosilada se basa en la medición de la cantidad de glucosa adherida a los glóbulos rojos y su resultado se expresa en porcentaje, que determina el nivel medio de glucemia durante el trimestre anterior a la prueba. Además, desde 2010, la American Diabetes Association estableció esta prueba como un exa- 
men de diagnóstico de la enfermedad: un resultado igual o superior a $6,5 \%$ determina que una persona tiene diabetes. (Jiménez, 2016)

Por otra parte Empendiúm (2018) también ha dejado en evidencia cierta controversia que se generó respecto a este indicador, a partir del momento en el que el Colegio Americano de Médicos (ACP, por sus siglas en inglés) con sede en los EE.UU. de América publicó sus recientes recomendaciones al respecto, que, en términos generales, refieren que se determinar el valor objetivo de la $\mathrm{HbA} 1 \mathrm{c}$ entre un $7 \%$ y un $8 \%$ y aplicar un tratamiento menos intenso cuando el nivel de $\mathrm{HbA} 1 \mathrm{c}<6,5 \%$. También proponen un tratamiento orientado a evitar los síntomas de la hiperglucemia o de la hipoglucemia en vez de dirigido hacia conseguir un valor determinado de $\mathrm{HbA} 1 \mathrm{c}$ en los grupos susceptibles. En estas poblaciones se engloban personas con afecciones crónicas severas (enfermedades renales, EPOC o insuficiencia cardíaca congestiva), con una esperanza de vida <10 años debido a la edad avanzada ( $\geq 80$ años), también aquellas ingresadas en residencias de ancianos y otras con un alto nivel de dependencia (como demencia o cáncer). (Empendium, 2018)

Es evidente que al menos con la primera las posiciones de la ACP se genera una disparidad respecto a lo estipulado por otras reconocidas organizaciones profesionales igualmente estadounidenses, tales como: la Asociación Americana de Diabetes (AAD, por sus siglas en inglés), quienes aconsejan un valor objetivo de $\mathrm{HbA} 1 \mathrm{c}$ de $<7 \%$; o también, la Asociación Estadounidense de Endocrinólogos Clínicos (AACE, por sus siglas en inglés) que por su parte ponderan un nivel de $\leq 6,5 \%$. Es por consiguiente que, de esa manera, se han generado importantes controversias y un difícil reto a los médicos quienes ahora tendrían que tratar de conciliar las recomendaciones provenientes de estas instituciones médicas.
Ahora bien, habiendo esclarecido cuales podrían las pruebas que, por lo general, se indican en la DM 2 y que bien pueden ser útiles para establecer caracterización hematológica, así como la importancia que más recientemente se ha desarrollado en torno al examen de $\mathrm{HbA} 1 \mathrm{c}$, se procede a referir algunos estudios recientes que se hallaron durante el presente proceso de revisión, debido a que en los mismos fue posible identificar algunas particularidades útiles para ejemplificar características hematológicas de pacientes con DM 2.

El primero de ellos es el de Osuna et al. (2014). Aunque los objetivos de ésta investigación están limitados a la caracterización demográfica y clínica de un grupo de 318 pacientes con DM 2 hospitalizados en el servicio de medicina interna del hospital de San José, Bogotá-Colombia, durante el periodo comprendido entre octubre de 2010 y agosto de 2012; se ha encontrado que, dentro de los datos clínicos considerados al momento de ingreso de los pacientes, fueron reportados los valores de glucosa y de hemoglobina glicosilada ( $\mathrm{HbA1c})$ que bien sirven de referencia para lo que se pretende dejar en evidencia. Entonces, según éstos tratadistas:

Al ingreso los niveles de glucosa registrados mínimo 12 mg/dL y máximo 1263 mg/ dL y mediana de 255 (RIQ 134-417).

[...] El nivel medio de HbA1c fue de $8.2 \pm$ 1.5 para los pacientes con diabetes conocida y $9.7 \pm 1.7$ en los diabéticos de novo. (pág. 347)

Entre varias de sus disertaciones, los mismos pudieron precisar que, mediante su propio estudio "el impacto del mal control ambulatorio podría estar en relación con los hallazgos al ingreso de la hospitalización, donde $81 \%$ se encontraban fuera de metas de control metabólico (49\% pobre control y $31.8 \%$ mal control)" (pág. 349) Más adelante también que se apoyan en las deduccio- 
nes de Greci et al. (como se ha citado en Osuna et al., 2014) ya que esos expertos igualmente aseguraron que "la medición del nivel de HbA1c para la detección de casos nuevos de diabetes, es de utilidad en los pacientes gravemente enfermos con hiperglucemia en el ingreso hospitalario" (pág. 349-350)

En otro estudio descriptivo realizado por Zerquera, Vicente, Rivas, \& Costa (2016), en el que se estimó conocer las características clínicas y la frecuencia de complicaciones crónicas en un grupo de 396 personas con diabetes mellitus tipo 2 ingresadas en el período de enero a diciembre del 2013 a un centro de atención especializado de Cienfuegos-Cuba, entre determinadas variables, se incluyó el análisis hematológico de los parámetros de la glucemia en ayunas, colesterol total, triglicéridos, ácido úrico, micro albuminuria. De los resultados obtenidos, se considera valioso rescatar que estos expertos indican que:

A pesar de que el consumo de energía de cada día para la población estudiada, se encontró dentro de las recomendaciones, la hiperglucemia que se presentó en este estudio, se puede explicar por la alimentación desequilibrada que se observó y el alto consumo de equivalentes correspondiente al grupo de azúcares, que puede explicar la presencia de este factor de riesgo, además del mayor consumo de grasas y aceites. Los valores de glucemia en ayunas de los pacientes incluidos en al[sic] investigación oscilan en los 14,0 $\mathrm{mmol} / \mathrm{l}$ aunque se debe tener en cuenta que muchos de estos pacientes son de reciente comienzo o diagnóstico de la enfermedad. (pág. 287)

El otro trabajo investigativo que sirve de referencia fue adelantado en 2018 por Alvarez,
Torres, Guadalupe, Delgado, \& Arrunategu. El objetivo de éste estudio observacional, de cohorte retrospectivo, fue el de reportar la existencia o no de diferencias hematológicas y bioquímicas entre un grupo de tres pacientes diagnosticados con diabetes mellitus tipo 2 (DM2) y otro grupo de tres pacientes sin DM 2, considerando que ambos grupos estuvieron bajo tratamiento de hemodiálisis (HD) entre diciembre de 2015 y diciembre de 2016 en la clínica CENPER de Lima, Perú. En muchos otros aspectos, tratadistas indicaron que:

Se obtuvieron los valores medios y
desviación estándar (DE) de cada
parámetro hematológico y bioquí-
mico durante 1 año (12 medicio-
nes en cada paciente). El contaje
completo de células sanguíneas
incluyó hemoglobina, hematocrito,
leucocitos, monocitos, eosinófilos,
basófilos, linfocitos y plaquetas.
[...]

Se recogieron valores trimestrales de proteínas totales (PT), albúmina plasmática (Alb) y colesterol total (CT); y mensuales de transferrina (Tr), dada su vida media más corta. Los parámetros bioquímicos también incluyo transaminasas TGP y TGO, triglicéridos, fosfatasa alcalina, colesterol de baja densidad (LDL), de alta densidad (HDL), proteína $C$ reactiva (PCR), calcio y fósforo en suero. El porcentaje de reducción de urea (PRU) fue calculado sustrayendo la concentración de urea inicial de la concentración de urea final y dividiéndola entre el porcentaje de urea inicial. (Alvarez et al., 2018, pág. 7)

De la misma manera, de sus resultados se pudo extraer que, por ejemplo, en el grupo de pacientes con DM 2, los parámetros hematológicos que más se diferenciaron fueron:

- Un reducido valor en la media del hematocrito de 28,5 $\pm 1,44 \%$ comparado con $34,3 \pm 1,55 \%$ en pacientes no diabéti-

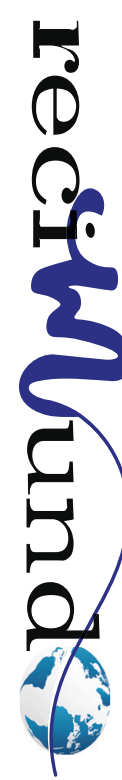


M. E.

$\cos (\mathrm{P}<0.05)$.

- Anemia que osciló de marcada a moderada (en ambos grupos), siendo de 8,9 $\pm 0,12 \mathrm{~g} / \mathrm{dl}$ en pacientes diabéticos.

- Una importante monocitosis (13,9 $\pm 1,82$ $\%)$, en comparación con los no diabéti$\cos (9,00 \pm 0,57 \%)(p<0.05)$.

- Menor conteo en el porcentaje promedio de linfocitos de 19,3 $\pm 1,01$, respecto al valor de 26,1 $\pm 0,31 \%$ en pacientes no diabéticos $(P<0.05)$.

- Una media del NLR significativamente alta, con un valor de 3,2 $\pm 0,38$; en comparación con la de pacientes no diabéticos de 2,3 $\pm 0,10$. $(P<0,05)$

- Elevados valores de PCR $(2,2 \pm 1,1)$ y transaminasa TGO $(18,9)$, en comparación con los 1,4 \pm 0,03 (de PCR) y 9,5 (de TGO) en pacientes no diabéticos.

En definitiva, éstos tratadistas resaltan que "los parámetros hematológicos tienen mayor significancia que los parámetros bioquímicos en la comparación de pacientes diabéticos y no diabéticos en HD" (Alvarez et al., 2018, pág. 9)

\section{Conclusiones}

Con base en las fuentes consultadas en el presente estudio, y considerando algunas limitaciones identificadas tras la aplicación de la metodología investigativa, como lo fue el muy escaso número de material bibliográfico en torno a la temática planteada, posiblemente por la rareza y especificidad de reporte de casos en los que se traten las características hematológicas de pacientes diagnosticados con DM 2; es posible atreverse a deducir que en pacientes con DM 2 es hematológicamente característico encontrar alteraciones, fundamentalmente, en los valores de: Insulina (elevada); Glucosa o Glucemia (hiperglucemia: elevada; o. hipoglucemia: disminuida), HbA1c (elevada), media de hematocritos (disminuida), recuento de monocitos (elevados), porcentaje promedio de linfocitos (reducido), PCR y transaminasas TGO (elevadas), colesterol
LDL (elevado), triglicéridos (elevados); entre otras variables menos frecuentes que, probablemente, pudieran estar alteradas de manera paerticular.

\section{Bibliografía}

Abbott. (2017). Diabetes - Guia de formacoión. Recuperado el 27 de Diciembre de 2020, de Sitio Web: Abbott Laboratories: https://www.corelaboratory.abbott/sal/learningGuide/ADD-00061643ES-EU\%20170098\%20Diabetes_Learning\%20 Guide.pdf

Alvarez, M., Torres, D., Guadalupe, H., Delgado, I., \& Arrunategui, V. (Julio-Septiembre de 2018). Características hematológicas y bioquímicas en pacientes con y sin diabetes mellitus tipo 2 (DM2) sometidos a hemodiálisis durante un año de seguimiento. Horizonte Médico, 18(3), 6-11. doi:10.24265/horizmed.2018.v18n3.02

Brutsaert, E. (Enero de 2019). Erika F. Brutsaert. Recuperado el 26 de Diciembre de 2020, de Sitio Web: Manual MDS: https://www.msdmanuals. com/es/professional/trastornos-endocrinol\%C3\%B3gicos-y-metab\%C3\%B3licos/diabetes-meIlitus-y-trastornos-del-metabolismo-de-los-hidratos-de-carbono/diabetes-mellitus-dm\#v988089_es

Empendium. (03 de Octubre de 2018). Diabetes mellitus tipo 2: diferentes valores objetivo para la HbA1c. Recuperado el 28 de Diciembte de 2020, de Sitio Web: Empendium: https://empendium.com/manualmibe/noticias/195762, diabetes-mellitus-tipo-2-diferentes-valores-objetivo-para-la-hba1c

Empendium. (2020). Manual de Medicina Interna Basada en la Evidencia. Recuperado el 26 de Diciembre de 2020, de Sitio Web: Empendium.com: https://empendium.com/manualmibe/chapter/ B34.II.13.1.

IDF. (2019). Atlas de la Diabetes de la FID (9a. ed., Vol. 9a. Edición). International Diabetes Federation. Recuperado el 26 de Diciembre de 2020, de https://diabetesatlas.org/upload/resources/material/20200302_133352_2406-IDF-ATLAS-SPANBOOK.pdf

Jiménez, C. (2016). ¿Qué es la hemoglobina glicosilada? Recuperado el 26 de diciembre de 2020, de Sitio Web: diabetes.ascensia.es/: https://www. diabetes.ascensia.es/blog/blog-detail-three/

Mayo Clinic - USA. (26 de Agosto de 2020). Diabetes de tipo 2. Recuperado el 26 de Diciembre de 2020, de Sitio Web: Mayo Clinic: https://www.mayoclinic.org/es-es/diseases-conditions/type-2-dia- 
betes/symptoms-causes/syc-20351193

MedlinePlus. (26 de Enero de 2020). Diabetes tipo 2. Recuperado el 26 de Diciembre de 2020, de Sitio Web: medlineplus.gov/spanish: https://medlineplus.gov/spanish/ency/article/000313.htm

MedlinePlus. (26 de Enero de 2020). Enciclopedia MédicalGlucosa en sanfre. (A.D.A.M. Inc.) Recuperado el 26 de Diciembre de 2020, de Sitio Web: MedlinePlus: https://medlineplus.gov/spanish/ ency/esp_imagepages/19825.htm

Ministerio de Salud Publica de Uruguay. (2017). Guía de práctica clínica de diabetes mellitus tipo 2 para la atención en el ámbito ambulatorio. Recuperado el 27 de Diciembre de 2020, de Sitio Web: Gobierno de Uruguay: https://www.gub.uy/ministerio-salud-publica/sites/ministerio-salud-publica/files/ documentos/publicaciones/Guia\%20Diabetes.pdf

NIDDK. (2016). Pruebas y diagnóstico de la diabetes. Recuperado el 26 de diciembre de 2020, de Sitio Web: niddk.nih.gov/: https://www.niddk.nih. gov/health-information/informacion-de-la-salud/ diabetes/informacion-general/pruebas-diagnosti$\mathrm{co}$

Osuna, M., Rivera, M., Bocanegra, C., Lancheros, A., Tovar, H., Hernández, J., \& Alba, M. (Octubre-Diciembre de 2014). Caracterización de la diabetes mellitus tipo 2 y el control metabólico en el paciente hospitalizado. Acta Medica Colombiana, 39(4), 234-351. Recuperado el 27 de Diciembre de 2020, de http://www.scielo.org.co/pdf/amc/v39n4/ v39n4a07.pdf
Parra, E., \& Martínez, J. (2019). Interpretación de los análisis en la diabetes mellitus. AMF - Actualización en Medicina de Familia, 15(2), 91-96. Recuperado el 26 de Diciembre de 2020, de https:// amf-semfyc.com/web/article_ver.php?id=2386

Suarez, J. (2019). Variabilidad de la hemoglobina glicosilada y frecuencia de enfermedad renal crónica en pacientes con diabetes mellitus tipo 2 con mal control glucémico en consulta ambulatoria de un hospital público de Perú. Universidad Peruana Cayetano Heredia, Facultad de Medicina. Lima: Universidad Peruana Cayetano Heredia. Recuperado el 26 de Diciembre de 2020, de http://190.116.48.43/bitstream/handle/upch/7723/ Variabilidad_SuarezRojas_Jacsel.pdf?sequen$\mathrm{ce}=1$ \&isAllowed $=\mathrm{y}$

Valer, Á. (Julio de 2020). Revisión bibliográfica sobre la diabetes mellitus tipo 2. Revista Electrónica de PortalesMedicos.com, 15(14), Página Inicial. Recuperado el 27 de Diciembre de 2020, de https:// www.revista-portalesmedicos.com/revista-medica/revision-bibliografica-sobre-la-diabetes-mellitus-tipo-2/

Zerquera, G., Vicente, B., Rivas, E., \& Costa, M. (Diciembre de 2016). Caracterización de los pacientes diabéticos tipo 2 ingresados en el Centro de Atención al Diabético de Cienfuegos. Revista Finlay, 6(4), 281-289. Recuperado el 27 de Diciembre de 2020, de http://scielo.sld.cu/pdf/rf/v6n4/ rf05406.pdf

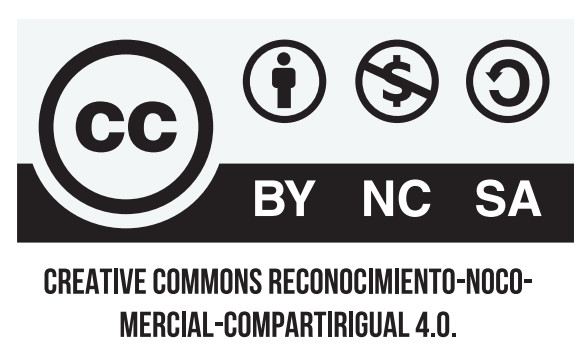

\section{CITAR ESTE ARTICULO:}

Campoverde Maldonado, M. J., Rosero Caiza, J. A., Rosero Caiza, G. E., \& Benavides Arteaga, M. E. (2021). Características hematológicas en pacientes con diabetes mellitus tipo 2. RECIMUNDO, 5(1), 20-31. https://doi. org/10.26820/recimundo/5.(1).enero.2021.20-31 